\title{
Anaesthesia and Postoperative Analgesia Performed by Insertion of a Perineural Catheter at the Brachial Plexus Site - Case Series
}

\author{
Lazar Alexandra, Szederjesi Janos*, Copotoiu Sanda Maria, Simon Noemi Szidonia, Badea ludita, \\ Azamfirei Leonard
}

University of Medicine and Pharmacy Tîrgu-Mures, Romania

\begin{abstract}
Postoperative pain management is of major importance and the existence of a device that ensures a good analgesia in the immediate postoperative period and also removes the side effects of the systemic drugs, is becoming a necessity. Objectives: The goal was to obtain a good quality anaesthesia and also a good postoperative analgesia by inserting a perineural catheter at the brachial plexus site. Material and method: This study included adult patients who underwent brachial plexus anaesthesia through a perineural catheter inserted at the brachial plexus site. The perineural catheter was introduced by ultrasound guidance with neurostimulation control. After insertion, a quantity of a anaesthetic admixture of $0.4 \mathrm{mg} / \mathrm{kg}$ is administered. The anaesthetic admixture contained Ropivacaine and Lidocaine, equimolar concentration of $0.5 \%$ In the postoperative period, the analgesia was ensured trough the already installed catheter. The analgesic mixture contained Ropivacaine and Lidocaine, equivalent concentrations of $0.25 \%$. The administration rate was $5 \mathrm{ml}$ every 4 hours, starting 6 hours postoperatively. Results: The anaesthesia, obtained through the perineural catheter, was a good quality anaesthesia ensuring both, good sensory and motor block. The feedback regarding postoperative analgesia was positive, this type of pain management being efficient and without the systemic drug side effects. This approach of brachial plexus block was accepted easily by the patients and was rated as a very satisfactory method. Conclusions: The insertion of a perineural catheter for anaesthesia and postoperative analgesia represents a safe and efficient method of achieving both analgesia and anaesthesia.
\end{abstract}

Keywords: anaestehsia, analgesia, perineural catheter, brachial plexus block, patient satisfaction

Received: 4 May 2015 / Accepted: 19 July 2015

\section{Introduction:}

For surgery of the hand both general and regional anaesthesia appear to satisfy the needs of analgesia and surgical comfort. Regional anaesthesia has some advantages over general anaesthesia. The most important seem to be: awareness and the sparing of major organs and systems of the body. Postoperatory, a performed brachial plexus block can provide analgesia for up to 8 hours, which is another important aspect [1].

There are several approaches for performing the brachial plexus blockade: supraclavicular, infraclavicular, interscalenic and axillary. The axillary approach is proved to be the safest due to the lowest risk of adjacent tissues lesions, frenic nerve blockade or pneumothorax, although the risk of intravascular injection persists [2].

Induced paresthesia and the transarterial approach were used to correctly locate the nerves for the brachial block. Peripheral neurostimulator introduction was salutary, helping for a precise location of the nervous structures. This method was the most used until ultrasounds were introduced as auxiliary locating method [3].

The possibility of visualization of the anatomical structures, of the anesthetic being administered and also of the needles among the nervous structures, raised the success

* Correspondence to: Janos Szederjesi

E-mail: yangzi37@gmail.com rate and reduced the incidents of the brachial plexus blockade when the ultrasounds were used $[1,4,5]$.

The perineural catheter (cPNB) was first described in 1946 , being used to stop a hiccup crisis. Since then it continued to develop, nowadays being used in a variety of procedures: vasospasm treatment in Raynaud disease [7] in vascular surgery and traumatology for increasing the blood flow in some ischemic areas [8] and in plastic surgery [9]. Most often, these catheters are used for postoperative pain management, the literature showing that this method is superior thanparenteral opioid administration [10].

\section{Objective}

The study aimed to analyze the quality of regional anesthesia performed by inserting a catheter at the brachial plexus site and also the quality of postoperative analgesia, realized through the same perineural catheter.

\section{Material and method}

We present a series of 3 cases, which are a part of a prospective, randomized study. The study received the approval of the Ethics Committee of the Clinical County Hospital of Tirgu-Mures no. 2987/2013 as well as the Ethics Committee of Tîrgu-Mureș University of Medicine and Pharmacy no.68/2014.

The inclusion criteria of our study were: age over 18 years, acute or chronic conditions of the hand with indica- 
tion for surgery and continuous hospitalization for at least 48 hours.

The exclusion criteria were: age under 18 years, allergies to the substances that were to be administered, psychiatric disorders, coagulation disorders, one day surgery and patient refusal to be enrolled.

Each patient filled in an informed consent form and after agreement to enter the study, they were monitored before and trough the anesthesia procedure: noninvasive blood pressure, electrocardiogram, peripheral oxygen saturation. A peripheral intravenous line was inserted before the procedure and the oxygen was supplemented by a face mask.

The patients were positioned supine and the affected limb with the forearm abducted at 900 . The elbow was flexed at 1100 . None of the patients received premedication.

The anesthetic procedure was performed by axillary approach and the anesthetic substance was administered through the inserted catheter. The anesthetized nerves were: median, ulnar, radial and musculocutaneus.

Weused theG\&ELogiqGeneralElectricechograph,USA9 $\mathrm{mm}$ linear probe and the Stimuplex (Bbraun, Melsugen, Germany) neurostimulator. The intensity of the electrical stimulation was between $0.3-0.5 \mathrm{~mA}$ for all the included patients.

For this study we used the ContiplexDSet- $400 \mathrm{~mm}$ (Bbraun, Melsugen, Germany) perineural catheters. The anesthetic drugs that were administered were Ropivacaine $1 \%$ and Lidocaine $1 \%$ in equimolar admixture of $0.5 \%$. The administered dose was $0.4 \mathrm{mg} / \mathrm{kgc}$.

The ultrasound probe was sterile draped and the procedure was started by locating the insertion site for the perineural catheter. After the correct image of the structures was obtained and after the right place of insertion was confirmed by neurostimulation, the stimulation needle is retrieved and the catheter is placed at the brachial plexus block. When the insertion procedure is completed the catheter is secured on the skin and the anesthetic is administered continuously and in slow injection.

Postoperatively, the analgesia was continued through the catheter by administering boluses of anesthetic admixture at every 4 hours,starting after 6 following surgery. The dose administered was 5 mililiters of a Ropivacaine and Lidocaine equimolar admixture of $0.25 \%-5 \mathrm{mg} / \mathrm{ml}$.

The information about the quality of the anesthesia and analgesia and also about the anesthesia side effects- nausea, vomiting, allergic reactionsor any other reactions were registered in a special designed form. Patients were also asked, before discharge, to fill a satisfaction form on a scale 1 to 5 where 5 was maximum satisfaction. The success of the anesthesia was assessed by thermic stimulation and also by response to pain.

It has to be noted that any other treatment of the patient, other than for postoperative pain, was carried as prescribed by the specialist. Alsoif the analgesia provided through the catheter was insufficient, the analgesia was completed with systemic drugs, opioids or others on doctor`s prescription.

\section{Case 1}

An adult man, 58 years of age, was admitted in emergency for post-traumatic total amputation of the left hand. Patient history was positive for: arterial hypertension, hepatic disease, gastric ulcer and renal disease.

After checking the informed consent, the monitoring and proper preparation of the site for the catheter insertion, the catheter was inserted with ultrasound guidance and neurostimulation control. It was secured on the skin and 45 milliliters from the admixture Ropivacaine and Lidocaine $0.5 \%$ were administered the equivalent of $225 \mathrm{mg}$ Lidocaine $0.5 \%$ and $225 \mathrm{mg}$ Ropivacaine $0.5 \%$.

The patient presented to surgery conscious and accepted the regional anesthesia- brachial plexus block with a perineural catheter insertion as his choice. After the anaesthesia was completed, he changed his mind and asked for general anaesthesia claiming the psychological comfort. Thus, we complied with the patient wish and we completed the regional anesthesia with general anesthesia by laryngeal mask and volatile anaesthetic- Sevoflurane.

After surgery, postoperative analgesia was provided only through the inserted perineural catheter, by administering 5 milliliters from the admixture Ropivacaine and Lidocaine $0.025 \%-5 \mathrm{mg} / \mathrm{ml}$, at every 4 hours, after six hours since the discharge from the operating room.

The perineural catheter was maintained for 72 hours. The patient was discharged with enteral analgesia. The degree of satisfaction was high, being graded with 5 in the satisfaction form.

\section{Case 2}

An adult male, 54 years of age accused pain on his left superior limb due to a digital nerve lesion is scheduled for neurorraphy. The patient consents to regional anesthesia and perineural catheter insertion. Through the inserted catheter 30 milliliters of the anesthetic admixture Ropivacaine and Lidocaine $0.5 \%$ were administered, which represents $175 \mathrm{mg}$ Lidocaine $0.5 \%$ and $175 \mathrm{mg}$ Ropivacaine $0.5 \%$.

A complete motor block was installed but the sensory was incomplete. Thus, we added another 5 milliliters of anesthetic admixture. After another 30 minutes, we obtained a complete brachial block.

After 4 hours since the surgery was finished, the patient complained of pain at the insertion site of the catheter. On visual examination we observe signs of inflammation on the skin surrounding the insertion site. We decided to remove the catheter and continue the analgesia

The degree of satisfaction was expressed regarding the anaestehsia only, and the patient graded as 5 the quality of anesthesia. Due to the fact that we removed the catheter before the postoperative analgesia was administered, the patient couldn't t express his opinion towards this matter. 


\section{Case 3}

An adult male, 53 years of age, presented to the operating theater after a crushing trauma on his left hand and open fractures and tendon lesions on second-third and fifth finger.

After the catheter was inserted at the brachial plexus site by axillary approach, we administered 30 milliliters of anesthetic admixture, representing $175 \mathrm{mg}$ Lidocaine $0.5 \%$ and $175 \mathrm{mg}$ Ropivacaine $0.5 \%$.

A complete motor block was obtained after 47 minutes, but an incomplete sensory bloc corresponding to the ulnar nerve. We supplemented the block with 5 milliliters of anesthetic. We achieved a complete sensory block in the following 15 minutes.

Postoperatory the analgesia was realized through the catheter with 5 milliliters from the anesthetic admixture Lidocaine and Ropivacaine $0.025 \%$ - every 4 hours. The catheter was removed 48 hours later, when the patient was discharged. No side effects were recorded. Patient $s$ satisfaction was graded with a 5 .

\section{Results}

Through the perineural catheter we achieved a good quality anesthesia which offered an optimal surgical comfort for both, the patients and the surgeon. All the studied patients had a superior quality of anesthesia.

The installation time was between 60-70 minutes, being necessary to supplement the anesthetic in 2 out of the three cases. This led to an optimal block for surgery.

Regarding the postoperative analgesia it was optimal for 2 out of the 3 cases presented. The third patient, due to the fact that presented an inflammatory reaction at the inserting site and we removed the catheter before any postoperative analgesia was administered, couldn't express his opinion on this method.

At the moment we present these results, the satisfaction degree is high, but the study is very limited due to its small number of enrolled patients.

The existence of a perineural catheter, through which we can administer analgesia continuously or in boluses removes the side effects of systemic analgesics, especially opioids.

The possibility of maintaining the catheter over 2 hours allows repeated surgical interventions without submitting the patient to the stress of a new anesthetic procedure.

\section{Discussions}

Analgesia in the immediate postoperative period is still largely studied, presenting the following advantages: in protocols for postoperative analgesia which should involve a perineural catheter; allows early mobilization of the operated limb, and increase patient comfort

The promote literature confers to perineural catheters a dominant role in pain management [11]. Thus, the anesthesia we obtained through the catheter was one of a good and placed this device in a good position for performing regional anesthesia. The weakness of this method was the prolonged time needed for anesthesia installation.

The procedure proved to be safe,we did not encounter any incidents during anesthesia such as vascular punctures or intravascular anesthetic injection. The high degree of safety is due to the ultrasound guidance that was used to position the catheter.

Postoperative analgesia was optimal being easy to conduct. We could avoid by this method the side effects of systemic analgesics, especially opioids which are listed as first line of postoperative pain management. The anesthetic admixture - Ropivacaine and Lidocaine 0,025\%- was reported to be a safe combination with a low toxicity degree [14].

For the postoperatory analgesia, we chose to administer the same admixture of equimolar anesthetics, only half the concentration used for anaesthesia. The reason was that we aimed to achieve only analgesia not anaesthesia and especially because one of a motor block. This is because one of our objectives was that the patient could mobilize his limb as soon as possible and without any pain.

Patients welcomed this method because they were spared of the multiple administrationof multiple drugs, each on different hours and with many side effects.

This study is very limited due to the small number of enrolled patients, aspect which impedes us to perform statistical analysis. With all this limitation we can only speculate that the insertion of a perineural catheter could offer both a good anesthesia and a good pain control in the postoperative period. Another advantage can be the opportunity to reuse the catheter for anaesthetic purposes if surgery is needed again.

This study represents the beginning of a new postoperatory pain management method, that was introduced in our hospital.

\section{Conclusions}

The insertion of a perineural catheter for regional anesthesia and postoperative pain management is a safe and efficient method of providing both anesthesia and analgesia.

The perineural catheter can become a standard method in regional anesthesia and analgesia, but more data is needed in order to complete this task.

\section{Acknowledgments}

This paper was published under the frame of European Social Found, Human Resources Development Operational Programme 2007-2013, project no. POSDRU/159/1.5/S/136893

\section{References}

1. Lazăr A, Szederjesi J, Azamfirei L, et al. Combination of Ropivacaine and Lidocaine for Long Lasting Locoregional Anesthesia. Acta Medica Marisiensis, 2014;60:41-43.

2. Ashish R.S, David M S. Axillary Brachial Plexus Block. Anesthesiol Res Pract, 2011; 2011: 173796.

3. Coventry DM, Barker KF, Thomson M. Comparison of two neurostimulation techniques for axillary brachial plexus blockade. $\mathrm{Br} \mathrm{J}$ Anaesth, 2001; 86:80-83. 
4. Kapral S, Greher M, Huber G, et al. Ultrasonographic guidance improves the success rate of interscalene brachial plexus blockade. RegAnest and Pain Med, 2008;33:253-258

5. Perlas A, Brull R, Chan WWS, McCartney CJL, Nuica A, Abbas S. Ultrasound guidance improves the success of sciatic nerve block at the popliteal fossa. Reg Anest and Pain Med, 2008;33:259-265.

6. ANSBRO FP. A method of continuous brachial plexus block. Am J Surg, 1946; 71:716-22.

7. Greengrass RA, Feinglass NG, Murray PM, Trigg SD. Continuous regional anesthesia before surgical peripheral sympathectomy in a patient with severe digital necrosis associated with Raynaud's phenomenon and scleroderma. Reg Anesth Pain Med, 2003; 28:354-358.

8. Vadivelu N, Gesquire M, Mitra S, et al. Safety of local anesthesia combined with monitored intravenous sedation for American Society of Anesthesiologists 3 and 4 patients undergoing lower limb-preservation procedures. Foot Ankle Surg, 2010;49:152-154.

9. Le-Wendling L, Enneking FK. Continuous peripheral nerve blockade for postoperative analgesia. Curr Opin Anaesthesiol, 2008;21:602-609.

10. Wu JJ, Lollo L, Grabinsky A. Regional anesthesia in trauma medicine. Anesthesiology Research and Practice, 2011;7:213- 281.

11. Richman JM, Liu SS, Courpas G, et al. Does continuous peripheral nerve block provide superior pain control to opioids? A meta-analysis. Anesth Analg, 2006;102:248-257.

12. Barr J, FraserL G, Puntillo Kathleen. Clinical practice guidelines for the management of pain,agitation, and delirium in adult patients in Intensive Care Unit. Crit Care Med, 2013; 41:263-306.

13. Feldman HS, Covino BG. Comparative motor-blocking effects of bupivacaine and ropivacaine: A new amino amide local anaesthetic, in the rat and dog. Anesth Analg, 1991;73:373-384. 